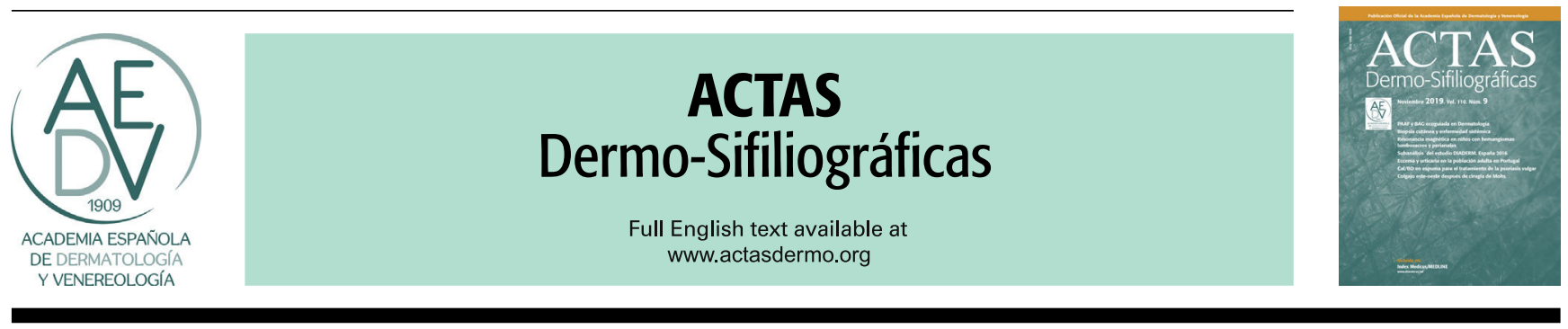

\title{
Validación del método de cuantificación del área corporal afectada por la psoriasis mediante lápiz óptico
}

\author{
A. Reolid ${ }^{a, *}$, O. Servitje ${ }^{b}, M$. Ginarte $^{c}$, A. Pérez-Ferriols ${ }^{d}, A$. Vélez $^{e}$, M. Aragües ${ }^{a}$, \\ M. Pereiro ${ }^{c}$, J.L. Sánchez-Carazo ${ }^{d}, J$. Garrido $^{f}$, E. Daudén ${ }^{a}$ \\ y Grupo de Trabajo de evaluación del Body Surface Area (BSA)
}

\footnotetext{
a Servicio de Dermatología, Hospital Universitario de la Princesa, Instituto de Investigación Sanitaria la Princesa (IIS-IP), Madrid, España

b Servicio de Dermatología, Hospital de Bellvitge, Barcelona, España

c Servicio de Dermatología, Complexo Hospitalario Universitario, Santiago de Compostela, España

' Servicio de Dermatología, Hospital General de Valencia, Valencia, España

e Servicio de Dermatología, Hospital Reina Sofía, Córdoba, España

${ }^{f}$ Departamento de Psicología Social y Metodología, Facultad de Psicología, Universidad Autónoma de Madrid, Madrid, España
}

Recibido el 8 de mayo de 2019; aceptado el 24 de julio de 2019

Disponible en Internet el 28 de agosto de 2019

\section{PALABRAS CLAVE \\ Psoriasis; \\ Superficie corporal; Body Surface Area; Escala medida; \\ Gravedad}

\begin{abstract}
Resumen
Introducción: La determinación de la superficie corporal afectada, Body Surface Area (BSA), es una de las escalas de medida más empleadas en la evaluación de la gravedad de la psoriasis, pero no está exenta de inconvenientes.

Objetivo: Validación de un nuevo sistema de medida del BSA.

Material y método: Estudio multicéntrico, prospectivo, que incluyó 56 pacientes con psoriasis. Cada paciente fue evaluado en 2 visitas por 2 dermatólogos del mismo Centro que valoraron BSA mediante 2 procedimientos: método visual «tradicional» $(M T)$, palma mano=1\%; y el método «lápiz óptico» (LO), lápiz capacitivo puntero sobre pantalla táctil con medición de la superficie mediante software específico.

Resultados: Se observó una concordancia aceptable entre ambos métodos, con coeficiente de correlación intraclase $(\mathrm{CCl})$ de 0,87 , pero con unos límites de acuerdo excesivamente grandes y un sesgo sistemático consistente en mayores medidas de BSA con MT que con LO. La concordancia entre métodos fue superior en el tronco y las extremidades inferiores $(\mathrm{CCl}>0,8)$. La fiabilidad intraobservador fue excelente con ambos métodos (CCI: MT, 0,97; LO, 0,98). La fiabilidad interobservador fue elevada (CCl: MT, 0,91; LO, 0,94), pero el BSA medio difirió significativamente entre observadores. Además, el CCI se redujo drásticamente cuando se consideró la cabeza exclusivamente.
\end{abstract}

\footnotetext{
* Autor para correspondencia.

Correo electrónico: alereolid@gmail.com (A. Reolid).
} 


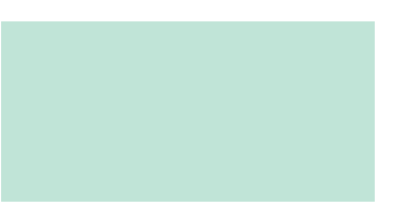

\section{KEYWORDS}

Psoriasis;

Body surface area;

Body Surface Area;

Measurement;

Severity

Conclusiones: El presente estudio valida el método LO para la medición de la superficie corporal afectada en pacientes con psoriasis. Muestra una buena concordancia con el MT, presentando menos variabilidad y mayor fiabilidad interobservador.

(c) 2019 AEDV. Publicado por Elsevier España, S.L.U. Este es un artículo Open Access bajo la licencia CC BY-NC-ND (http://creativecommons.org/licenses/by-nc-nd/4.0/).

\section{Validation of an Optical Pencil Method to Estimate the Affected Body Surface Area} in Psoriasis

\section{Abstract}

Introduction: Body surface area (BSA) affected by psoriasis is one of the most often used measures for assessing severity, but this method has shortcomings.

Objective: To validate a new way to estimate BSA.

Material and method: Prospective, multicenter study in 56 patients with psoriasis. Each patient was evaluated by 2 dermatologists in 2 visits to the same hospital. Each dermatologist used 2 methods for estimating BSA: the traditional visual estimation in which the area of the palm equals $1 \%$ of the total body surface and an optical pencil (OP) method in which the affected area is drawn on a touch screen. Software in the application then calculates the BSA.

Results: Overall concordance between the 2 methods was acceptable according to an intraclass correlation coefficient (ICC) of 0.87 . However, the limits of agreement were unacceptably large and there was systematic bias: traditional estimates were consistently greater than OP calculations. Concordance between the methods was better (ICC $>0.8)$ on the trunk and lower extremities. Intraobserver reliability was excellent with both methods (ICCs, 0.97 and 0.98 for the traditional and OP estimates, respectively). Interobserver reliability was also high (ICCs, 0.91 and 0.94 for the traditional and OP methods), although the mean BSA differed significantly between observers. The ICCs were much lower for BSA estimates on the head.

Conclusions: This study to validate the OP method for estimating the affected BSA in patients with psoriasis shows good agreement between the OP and traditional approaches. The OP calculations also showed less variance and better interobserver reliability.

(C) 2019 AEDV. Published by Elsevier España, S.L.U. This is an open access article under the CC BY-NC-ND license (http://creativecommons.org/licenses/by-nc-nd/4.0/).

\section{Introducción}

La psoriasis es una enfermedad inflamatoria que afecta al 2-3\% de la población adulta en Europa y Estados Unidos ${ }^{1}$, ocasionando un considerable deterioro de la calidad de vida $^{2,3}$. Actualmente existe cierta controversia a la hora de clasificar la gravedad de la psoriasis en 2 (leve y moderada-grave) o en 3 formas (leve, moderada y grave) (-6 $6^{\text {. }}$. Se han empleado diferentes escalas para evaluar de una manera objetiva su gravedad, aunque ninguna satisface plenamente a la comunidad internacional ${ }^{7,8}$. El Psoriasis Area and Severity Index (PASI), con una elevada fiabilidad intere intraobservador ${ }^{9}$, es el más empleado y aceptado a pesar de tener conocidas limitaciones ${ }^{5}$. Con objeto de tener en consideración la valoración que el propio paciente hace de su enfermedad, se utiliza el Dermatology Life Quality Index (DLQI), un cuestionario de calidad de vida cuya falta de equivalencia transcultural limita su validez. La tercera escala más empleada es el Body Surface Area (BSA). El método clásico (o tradicional) de evaluación consiste en la determinación visual de la superficie corporal afectada considerando que la palma del paciente que está siendo evaluado supone el $1 \%$ de la superficie corporal. Por tanto, puede ir del 0 al $100 \%$. La superficie corporal se subdividiría en cabeza y cuello $(9 \%)$, cada miembro superior $(9 \%)$, cara anterior (18\%) y posterior $(18 \%)$ del tronco, cada miembro inferior $(18 \%)$ y periné $(1 \%)^{10}$. En concordancia con otros grupos internacionales y recomendaciones de expertos ${ }^{4,11,12}$, el Grupo de Psoriasis de la Academia Española de Dermatología y Venereología alcanzó el consenso de definir la gravedad de la psoriasis en función de diferentes criterios, entre los que se incluye el PASI, el BSA y el DLQI evaluados por la misma persona ${ }^{2}$.

El BSA resulta subjetivo, tiende a la sobreestimación, es poco útil en algunas formas clínicas (p. ej., psoriasis en gotas), es difícil de valorar en pacientes con discromía residual y la regla del $1 \%$ resulta controvertida ${ }^{12}$.

El objetivo del estudio es validar un sistema de software de evaluación del BSA («lápiz óptico»), comparándolo con el método tradicional. La hipótesis de trabajo es que el software podría suponer una evaluación más objetiva del BSA que permitiera reducir la variabilidad inter- e intraobservador.

\section{Pacientes y método}

Se realizó un estudio multicéntrico, prospectivo, en el que se incluyeron pacientes mayores de edad, con psoriasis en placas, que acudieron de forma consecutiva a diferentes Centros hospitalarios en España y Portugal (Hospital Universitario de la Princesa en Madrid, Hospital General 


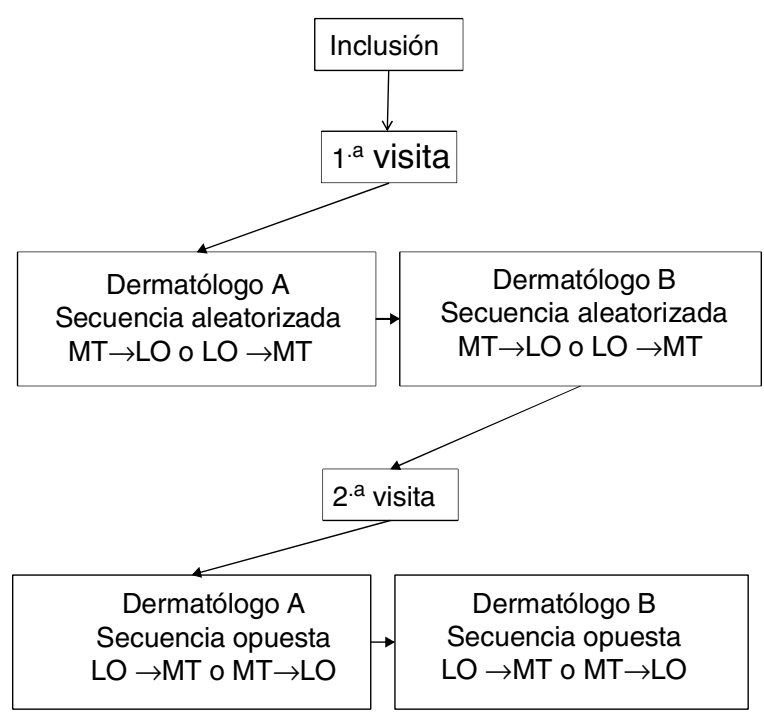

Figura 1 Diseño del estudio. LO: método del lápiz óptico; MT: método tradicional.

de Valencia, Hospital de Bellvitge de Barcelona, Complexo Hospitalario Universitario de Santiago de Compostela, Hospital Reina Sofía de Córdoba y Hospital Santa María de Lisboa). El estudio contó con la aprobación de los Comités Éticos de cada Centro, así como del consentimiento informado y escrito de cada paciente.

Cada paciente fue evaluado en 2 visitas, espaciadas $3 \pm 1$ días. En la primera visita se recogieron variables sociodemográficas y clínicas. En cada visita, 2 dermatólogos del mismo Centro, ciegos a los resultados del otro dermatólogo, realizaban la valoración de la superficie corporal afectada mediante el método «tradicional» (MT) y el software «lápiz óptico» (LO) diseñado por Serono ${ }^{\circledR}$. No debía transcurrir más de una hora entre la valoración de los investigadores. La figura 1 muestra el diseño experimental.

Para utilizar el software LO, el investigador aplicaba un lápiz capacitivo puntero sobre una pantalla táctil con un diagrama representando la figura humana y sombreaba las zonas afectadas, obteniendo una planimetría completa de la superficie corporal lesionada (fig. 2), almacenando dicha información en el software. El orden de aplicación de los métodos de evaluación del BSA (MT $\rightarrow$ LO o LO $\rightarrow$ MT) se aleatorizó previamente a la primera visita y se invirtió en la segunda.

Estadística. Las medidas de BSA se resumen como media y desviación típica (DT). Para estudiar la concordancia entre métodos se utilizó el coeficiente de correlación intraclase $(\mathrm{CCl})$ de efectos mixtos para acuerdo absoluto ${ }^{13}$. Este mismo modelo se utilizó en todos los análisis excepto para el estudio de la fiabilidad interobservador, para el que se utilizó el $\mathrm{CCl}$ de un factor para acuerdo absoluto. Además se utilizó el método gráfico de Bland-Altman ${ }^{14}$ para estudiar la diferencia entre métodos, $d$, la DT de la diferencia, $s$, los límites de acuerdo, $d-1,96 s$ y $d+1,96 s$, y las diferencias individuales entre métodos. Para comparar la variabilidad de ambos métodos se utilizó la prueba de Levene de igualdad de varianzas ${ }^{15}$. Finalmente, se realizó un análisis de varianza (ANOVA) con 4 factores intrasujetos: método, investigador, visita y orden de aplicación. Se estudiaron los efectos principales y las interacciones de $1 .^{\circ}, 2 .^{\circ}$ y $3 .^{\text {er }}$ orden.

Se utilizó un cuestionario con preguntas dicotómicas cerradas para evaluar las opiniones de los observadores sobre el método LO, incluyendo preguntas sobre factibilidad, utilidad como herramienta de prescripción, posible estandarización, utilidad en estudios clínicos y utilidad en práctica clínica.

Se consideraron significativos valores de $p<0,05$. El análisis estadístico fue realizado con el paquete SPSS 21.0.

\section{Resultados}

Se incluyeron 56 pacientes, 34 hombres $(60,7 \%)$ y 22 mujeres $(39,3 \%)$ con edad media (DT) de 49,93 $(16,76)$ años. El tiempo medio de evolución de la psoriasis desde el diagnóstico fue de $18,77(14,28)$ años. El PASI basal medio fue de $11,4(8,7)$. Los tratamientos administrados eran: tópicos, 24 pacientes $(42,9 \%)$; fototerapia, $17(30,4 \%)$; terapia sistémica convencional, 14 (25\%), y terapia biológica, 16 (28,6\%).
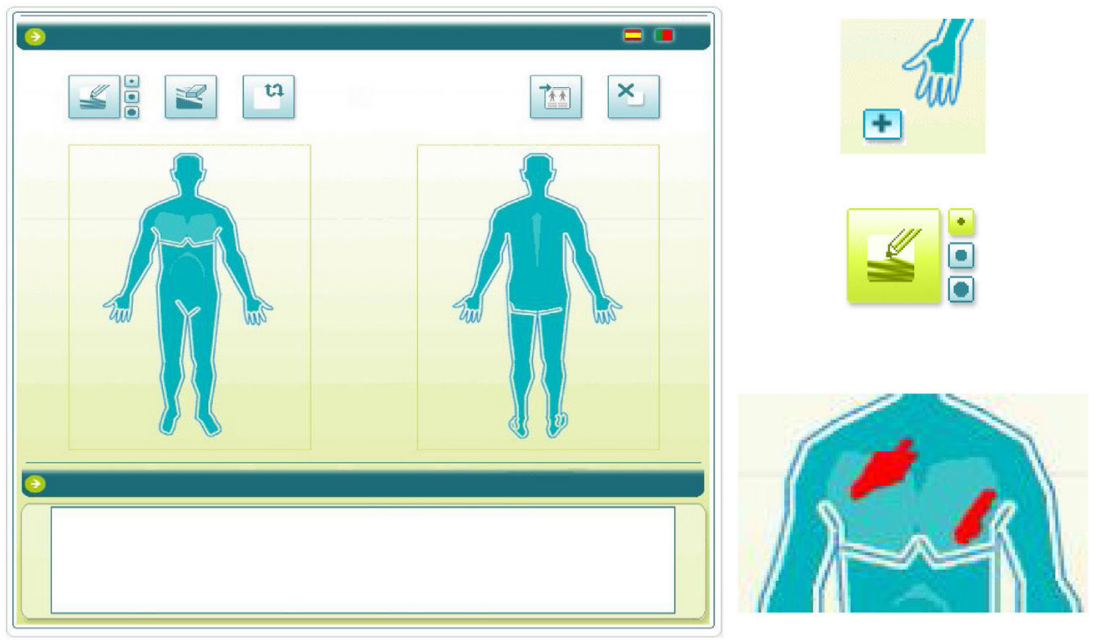

Figura 2 Detalles del método del lápiz óptico. 
Tabla 1 Body Surface Area (BSA) medio de 4 determinaciones en 56 pacientes $(\mathrm{N}=224)$ con los métodos tradicional y lápiz óptico para todo el cuerpo y por zonas analizadas. Coeficiente de correlación intraclase $(\mathrm{CCl})$ entre los métodos

\begin{tabular}{|c|c|c|c|c|c|}
\hline \multirow[b]{2}{*}{ Zona } & \multicolumn{2}{|c|}{ Método } & \multirow[b]{2}{*}{$\begin{array}{l}\text { Diferencia } \\
\text { Media (DT) }\end{array}$} & \multirow[b]{2}{*}{$\mathrm{p}$} & \multirow[b]{2}{*}{$\mathrm{CCl}(\mathrm{IC} 95 \%)$} \\
\hline & $\begin{array}{l}\text { Tradicional } \\
\text { Media (DT) }\end{array}$ & $\begin{array}{l}\text { Lápiz óptico } \\
\text { Media (DT) }\end{array}$ & & & \\
\hline El cuerpo como un todo & $25,63(20,90)$ & $19,36(19,50)$ & $6,27(8,86)$ & $<0,001$ & $0,87(0,83 ; 0,90)$ \\
\hline Cabeza & $1,49(2,15)$ & $0,79(1,19)$ & $0,70(1,49)$ & $<0,001$ & $0,57(0,47 ; 0,65)$ \\
\hline Tronco & $8,29(8,37)$ & $6,97(7,99)$ & $1,32(4,18)$ & 0,011 & $0,86(0,82 ; 0,89)$ \\
\hline Extremidades superiores & $4,42(4,21)$ & $3,13(3,63)$ & $1,29(2,55)$ & $<0,001$ & $0,74(0,68 ; 0,80)$ \\
\hline Extremidades inferiores & $9,41(8,24)$ & $7,94(7,93)$ & $1,47(4,23)$ & 0,006 & $0,85(0,81 ; 0,88)$ \\
\hline
\end{tabular}

Los datos se presentan como media y desviación típica (DT).

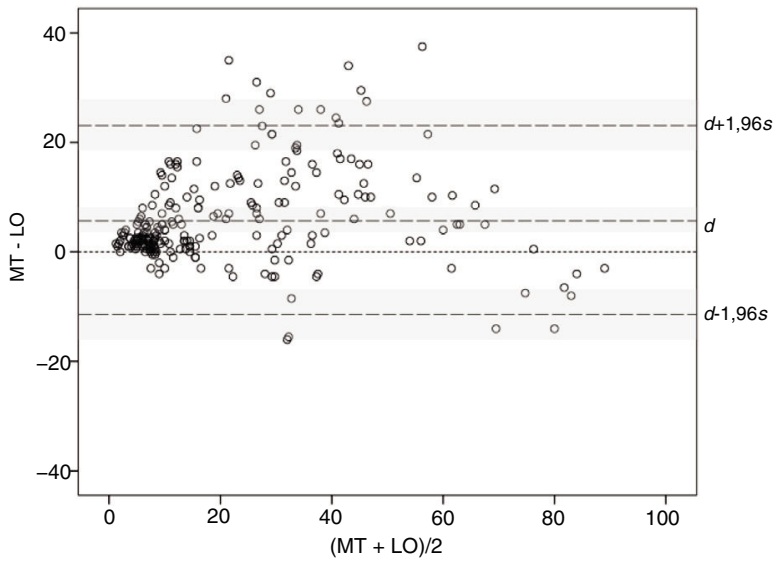

Figura 3 Concordancia gráfica entre métodos. $d$ : diferencia media; LO: método del lápiz óptico; MT: método tradicional; s: desviación típica de la diferencia.

\section{Concordancia entre métodos de medición}

Con el método MT, el valor medio (DT) del BSA obtenido en 224 determinaciones (4 determinaciones $\times 56$ pacientes) fue de 25,63 $(20,90)$, significativamente mayor que con el LO, que fue de $19,36(19,5)(p<0,001)$, y lo mismo sucedió en las diferentes zonas analizadas (tabla 1$)$.

El CCl entre ambos métodos fue de 0,87 (IC95\%: 0,83$0,90)$. Por zonas analizadas, los $\mathrm{CCl}$ mayores se encontraron en el tronco y las extremidades inferiores, siendo menor en las extremidades superiores y aún menor en la cabeza.

Se ha estudiado la concordancia entre métodos utilizando el método gráfico de Bland-Altman. La diferencia media 5,85 y su intervalo de confianza del $95 \%$ (IC95\%: 3,94-8,59) (señaladas como $d$ y la zona sombreada alrededor de esta en la figura 3) quedan íntegramente por encima del valor 0 , confirmando la existencia de un sesgo sistemático, consistente en que las medidas de BSA son mayores con el MT que con el LO. Además, la distribución de las diferencias sugiere que el sesgo se produce con valores de BSA pequeños $y$ medianos, donde el BSA con el MT es frecuentemente mayor que el obtenido con el LO, mientras que con los valores muy grandes, BSA $>70$, se invierte la tendencia y la medida con el LO resulta mayor que con el MT. Por otra parte, los límites de acuerdo, límite inferior $-11,10$ y límite superior 23,55 (señalados como $d-1,96 s$ y $d+1,96$ s con sus respectivos IC95\% [zonas sombreadas] en la figura 3), resultan algo mayores de lo deseable, sugiriendo una precisión moderada en alguno de los métodos.

\section{Concordancia intermétodos en cada visita}

Se ha estudiado la concordancia entre ambos métodos en cada una de las visitas, utilizando las 2 mediciones por paciente y método realizadas en cada visita $(\mathrm{N}=112)$. Los BSA medios (DT) para MT y LO en la primera visita fueron $26,35(21,66)$ y $20,14(17,78)$, respectivamente $(p<0,001)$; y para la segunda visita, $24,91(20,21)$ y $19,43(19,34)$, respectivamente $(p<0,001)$. En ambas visitas el BSA medio fue mayor con el MT que con el LO.

$\mathrm{El} \mathrm{CCl}$ entre los 2 métodos para aquellos pacientes en quienes se realizó la primera evaluación del BSA según la secuencia $M T \rightarrow$ LO $(N=56)$ fue de 0,87 (IC95\%: 0,82-0,91) y para aquellos pacientes evaluados en la secuencia inversa $(\mathrm{N}=56)$ fue de 0,86 (IC95\%: 0,80-0,90). La concordancia entre métodos en cada visita fue similar a la concordancia global.

\section{Fiabilidad test-retest con cada método}

Los BSA medios (DT) de 2 determinaciones $\times 56$ pacientes $(N=112)$ en la primera y segunda visita para el $M T$ fueron de $25,56(21,01)$ y $24,91(20,21)$, respectivamente $(p=0,128)$; y para el LO, de $19,38(19,13)$ y $19,43(19,34)$, respectivamente $(p=0,536)$. No se encontraron diferencias significativas entre los BSA medios obtenidos en los días 1 y 3 con ninguno de los 2 métodos. La tabla 2 ofrece los ICC y sus IC95\% entre la primera y segunda visita para cada método en el cuerpo como un todo y en cada zona estudiada. Los $\mathrm{CCl}$ obtenidos fueron mayores para el LO que para el MT en el cuerpo como un todo y en las 4 zonas anatómicas consideradas.

\section{Concordancia interobservador con cada método}

Los BSA medios (DT) para el primer (A) y segundo (B) observador para el MT $(\mathrm{N}=112)$ fueron de $24,35(20,48)$ y $26,92(21,36)$, respectivamente $(p=0,012)$; y para el $L O$, de $18,65(18,61)$ y $20,92(20,42)$, respectivamente $(p=0,004)$, sugiriendo que las valoraciones del observador $B$ fueron superiores a las del observador $A$ independientemente del procedimiento utilizado. La tabla 3 ofrece los ICC y sus 
Tabla 2 Coeficiente de correlación intraclase $(\mathrm{CCl})$ entre visitas $(\mathrm{N}=112)$ para cada método de valoración del Body Surface Area (BSA), global por zonas corporales

\begin{tabular}{llr}
\hline Zona & $\begin{array}{l}\text { Método tradicional } \\
\text { CCI (IC95\%) }\end{array}$ & $\begin{array}{l}\text { Lápiz óptico } \\
\text { CCI (IC95\%) }\end{array}$ \\
\hline El cuerpo como un todo & $0,975(0,963 ; 0,982)$ & $0,985(0,980 ; 0,990)$ \\
Cabeza & $0,789(0,710 ; 0,850)$ & $0,870(0,820 ; 0,910)$ \\
Tronco & $0,847(0,780 ; 0,890)$ & $0,971(0,960 ; 0,980)$ \\
Extremidades superiores & $0,872(0,820 ; 0,910)$ & $0,952(0,930 ; 0,970)$ \\
Extremidades inferiores & $0,909(0,870 ; 0,940)$ & $0,968(0,960 ; 0,980)$
\end{tabular}

Tabla 3 Coeficiente de correlación intraclase $(\mathrm{CCl})$ entre observadores para cada método de valoración del Body Surface Area (BSA) y área corporal considerada

\begin{tabular}{lll}
\hline Zona & $\begin{array}{l}\text { Método tradicional } \\
\text { CCI (IC95\%) }\end{array}$ & $\begin{array}{l}\text { Lápiz óptico } \\
\text { CCI (IC95\%) }\end{array}$ \\
\hline El cuerpo como un todo & $0,914(0,880 ; 0,940)$ & $0,945(0,920 ; 0,960)$ \\
Cabeza & $0,628(0,50 ; 0,73)$ & $0,766(0,67 ; 0,84)$ \\
Tronco & $0,895(0,85 ; 0,93)$ & $0,932(0,90 ; 0,95)$ \\
Extremidades superiores & $0,857(0,80 ; 0,90)$ & $0,896(0,85 ; 0,93)$ \\
Extremidades inferiores & $0,825(0,75 ; 0,88)$ & $0,848(0,78 ; 0,89)$ \\
\hline
\end{tabular}

IC95\% entre los observadores A y B para cada método en el cuerpo como un todo y en cada una de las áreas estudiadas. Independientemente del área considerada, los $\mathrm{CCl}$ fueron mayores para el LO que para el MT. La zona corporal que mostró una menor concordancia entre los dermatólogos en ambos métodos fue la cabeza.

\section{Análisis de la varianza}

La prueba de Levene sobre la igualdad de varianzas demostró que la varianza de BSA medida con LO era significativamente inferior $(p<0,001)$ que con el MT.

El ANOVA de medidas repetidas tan solo encontró un efecto principal del factor método $\left(F_{1}, 431=8,742\right.$; $p=0,003)$. Ninguno de los restantes efectos principales ni las interacciones estudiadas alcanzaron significación estadística.

\section{Opinión de los investigadores sobre el método «lápiz óptico»}

La mayoría de los 12 investigadores consideraron que el método LO era una herramienta fácil de usar (92\%), que debería estar estandarizada (83\%) y debería emplearse en estudios clínicos (100\%), mientras que recibió menos apoyo su utilización para la prescripción (67\%) y la práctica clínica diaria $(50 \%)$.

\section{Discusión}

A pesar de que existe acuerdo entre los dermatólogos sobre la necesidad de emplear escalas de medición en la práctica clínica, la realidad es que raramente se emplean de forma rutinaria. La valoración tradicional del BSA está basada en la asunción subjetiva de la regla del $1 \%{ }^{13}$, reduciendo la fiabilidad interobservador, convirtiéndola en una forma de evaluación no exenta de posibles errores ${ }^{4,8}$. Aunque se han hecho muchos avances para automatizar la medición del $\mathrm{PASI}^{16-19}$, son mucho menores las investigaciones orientadas a conseguir una evaluación más objetiva del BSA.

Se ha elaborado un sistema mediante el cual la señalización de la superficie afectada en un diagrama de una pantalla táctil con un puntero digital determina cuantitativamente el porcentaje de área afectada: método del LO.

Se observó una concordancia aceptable entre el MT y el LO, aunque en un rango amplio de valores de BSA el MT tiende a arrojar medidas mayores que el LO, hallazgo congruente con los del metaanálisis de Rhodes et al. ${ }^{12}$, demostrando que el BSA basado en la regla del 1\% sobreestima el verdadero BSA en adultos. Por otra parte, el límite de acuerdo superior estimado en el gráfico de Bland y Altman, $23,55 \%$, resulta grande, lo que sugiere falta de precisión de alguno de los métodos ${ }^{14}$, hallazgo congruente con los de algunos autores ${ }^{15,20}$, que sugieren que el MT es poco preciso y reproducible. Ambos métodos muestran una concordancia superior en zonas grandes como el tronco y las extremidades inferiores frente a zonas pequeñas como la cabeza y las extremidades superiores, donde la concordancia es peor.

Puesto que en cada visita la segunda medida de cada observador no era ciega a la primera medida, no se puede descartar un efecto de carry-over. Por esta razón la secuencia de aplicación de los métodos se aleatorizó en la primera visita y se invirtió en la segunda. Los CCl obtenidos en ambas secuencias fueron similares entre sí, sugiriendo que no se produjo un efecto de carry-over.

Respecto a la fiabilidad test-retest, se ha medido la concordancia entre las evaluaciones de la primera y la segunda visita, mostrando unos $\mathrm{CCl}$ superiores a 0,95 para el cuerpo como un todo. La fiabilidad test-retest para el LO es también excelente para las zonas tronco, extremidades inferiores y extremidades superiores, siendo ligeramente inferior para 
la cabeza. Estos resultados indican una excelente fiabilidad test-retest.

Ramsay y Lawrence ${ }^{21}$ comunicaron BSA medios en una muestra de 10 pacientes entre el 14 y el 33\% en función del observador. Más recientemente, otros autores ${ }^{8,22,23}$ encontraron una modesta fiabilidad interobservador del BSA. En nuestros resultados hemos encontrado diferencias significativas entre observadores en el BSA medio obtenido por cualquiera de los 2 métodos. Sin embargo, la fiabilidad interobservador fue buena con ambos métodos, con $\mathrm{CCl}$ mayores para LO que para MT, tanto para el cuerpo como un todo como en varias zonas estudiadas, aunque los valores de $\mathrm{CCl}$ cayeron drásticamente cuando se consideró exclusivamente la cabeza.

Por último, en cuanto a las opiniones de los dermatólogos participantes, la mayoría consideró que el método del LO era una herramienta de fácil manejo, estandarizable para evaluar el área corporal afectada, y que podría ser útil para la investigación clínica. Adicionalmente, el LO favorecería el control evolutivo de los pacientes al facilitar el almacenamiento informático de los datos, que podrían incorporarse a las historias clínicas electrónicas.

Una importante limitación de este estudio es que no se dispone de un archivo fotográfico que permitiría establecer un patrón oro con el que comparar los hallazgos con cada una de las técnicas utilizadas. Otra limitación supone la posible subestimación del BSA con el método del LO al transformar una topografía 3D en una proyección $2 \mathrm{D}$, en comparación con el MT. Tampoco se dispone de una medida formal del tiempo utilizado para cada uno de los métodos, aunque es opinión de los investigadores que el tiempo dedicado es aproximadamente el mismo, o incluso inferior pues se obvia el cálculo mental que debe realizar el investigador con el MT.

En conclusión, el presente estudio valida el método LO para la medición del BSA en pacientes con psoriasis. La concordancia con el MT no es tan buena como cabría esperar de métodos intercambiables, pero ello es posiblemente debido a las limitaciones del MT. Por otra parte, la fiabilidad testretest fue excelente y la fiabilidad interobservador mejoró discretamente respecto al MT. El LO es un método fiable que, si bien podría aplicarse en la práctica clínica diaria, va dirigido especialmente a su uso en estudios de investigación y ensayos clínicos.

\section{Conflicto de intereses}

Los autores declaran no tener ningún conflicto de intereses.

\section{Bibliografía}

1. Christophers E. Psoriasis-epidemiology and clinical spectrum. Clin Exp Dermatol. 2001;26:314-20.

2. Dauden E, Puig L, Ferrandiz C, Sanchez-Carazo JL, HernanzHermosa JM. Consensus document on the evaluation and treatment of moderate-to-severe psoriasis: Psoriasis Group of the Spanish Academy of Dermatology and Venereology. J Eur Acad Dermatol Venereol. 2016;30 Suppl. 2:1-18.

3. Finlay AY. Current severe psoriasis and the rule of tens. $\mathrm{Br} \mathrm{J}$ Dermatol. 2005;152:861-7.
4. Jose RM, Roy DK, Vidyadharan R, Erdmann M. Burns area estimation-an error perpetuated. Burns. 2004;30:481-2.

5. Kimball AB, Jacobson C, Weiss S, Vreeland MG, Wu Y. The psychosocial burden of psoriasis. Am J Clin Dermatol. 2005;6:383-92.

6. Llamas-Velasco M, de la Cueva P, Notario J, Martínez-Pilar L, Martorell A, Moreno-Ramírez D. Moderate psoriasis: A proposed definition. Actas Dermosifiliogr. 2017;108:911-7.

7. Krueger GG, Feldman SR, Camisa C, Duvic M, Elder JT, Gottlieb $A B$, et al. Two considerations for patients with psoriasis and their clinicians: what defines mild, moderate, and severe psoriasis? What constitutes a clinically significant improvement when treating psoriasis? J Am Acad Dermatol. 2000;43 2 Pt $1: 281-5$.

8. Puzenat E, Bronsard V, Prey S, Gourraud PA, Aractingi S, Bagot $M$, et al. What are the best outcome measures for assessing plaque psoriasis severity? A systematic review of the literature. J Eur Acad Dermatol Venereol. 2010;24 Suppl. 2:10-6.

9. Lebwohl M. A clinician's paradigm in the treatment of psoriasis. J Am Acad Dermatol. 2005;53 Suppl. 1:S59-69.

10. Long CC, Finlay AY, Averill RW. The Rule of Hand: 4 hand areas $=2$ $\mathrm{FTU}=1 \mathrm{~g}$. Arch Dermatol. 1992;128:11-30.

11. Mrowietz U, Kragballe K, Reich K, Spuls P, Griffiths CE, Nast A, et al. Definition of treatment goals for moderate to severe psoriasis: a European consensus. Arch Dermatol Res. 2011;303:1-10.

12. Rhodes J, Clay C, Phillips M. The surface area of the hand and the palm for estimating percentage of total body surface area: Results of a meta-analysis. Br J Dermatol. 2013;169:76-84.

13. Koo T, Li M. A guideline of selecting and reporting intraclass correlation coefficients for reliability research. J Chiropr Med. 2016;15:155-63.

14. Bland J, Altman D. Statistical methods for assessing agreement between two methods of clinical measurement. Lancet. 1986;327:307-10.

15. Levene H. Robust tests for the equality of variances. En: Olkins FJ, editor. Contributions to probability and statistics. Palo Alto, CA: Standford University Press; 1960.

16. Fink C, Alt C, Uhlmann L, Klose C, Enk A, Haenssle HA. Precision and reproducibility of automated computer-guided Psoriasis Area and Severity Index measurements in comparison with trained physicians. Br J Dermatol. 2019;180:390-6.

17. Fink C, Fuchs T, Enk A, Haenssle HA. Design of an algorithm for automated computer-guided PASI measurements by digital image analysis. J Med Syst. 2018;42:248.

18. Fink C, Uhlmann L, Klose C, Haenssle HA. Automated, computerguided PASI measurements by digital image analysis versus conventional physicians' PASI calculations: study protocol for a comparative, single-centre, observational study. BMJ Open. 2018;8:e018461.

19. Kreft S, Kreft M, Resman A, Marko P, Kreft KZ. Computer-aided measurement of psoriatic lesion area in a multicenter clinical trial-comparison to physician's estimations. J Dermatol Sci. 2006;44:21-7.

20. Henseler T, Schmitt-Rau K. A comparison between BSA, PASI PLASI and SAPASI as measures of disease severity and improvement by therapy in patients with psoriasis. Int J Dermatol. 2008;47:1019-23.

21. Ramsay B, Lawrence CM. Measurement of involved surface area in patients with psoriasis. Br J Dermatol. 1991;124:565-70.

22. Bozek A, Reich A. The reliability of three psoriasis assessment tools: Psoriasis area and severity index, body surface area and physician global assessment. Adv Clin Exp Med. 2017;26:851-6.

23. Yune YM, Park SY, Oh HS, Kim DJ, Yoo DS, Kim IH, et al. Objective assessment of involved surface area in patients with psoriasis. Skin Res Technol. 2003;9:339-42. 